REZENSION ZU: REIMER, DANIELA (HRSG.) (2019): SOZIALPÄDAGOGISCHE BLICKE. WEINHEIM/BASEL: BELTZ JUVENTA, 316 SEITEN, ISBN: 978-3-7799-6123-9, 34,95 EURO

Rebecca Mörgen

Universität Zürich, Institut für Erziehungswissenschaft

E-Mail: rmoergen@ife.uzh.ch

URL: https://www.ife.uzh.ch/de/research/abe/mitarbeitende2/moergenrebecca.html

Zitationsvorschlag:

Mörgen, Rebecca (2020): Rezension zu: Reimer, Daniela (Hrsg.) (2019): Sozialpädagogische Blicke. Weinheim/Basel: Beltz Juventa. In: Gesellschaft - Individuum - Sozialisation (GISo). Zeitschrift für Sozialisationsforschung 1 (1). DOI: 10.26043/GISo.2020.1.6

Link zum Artikel:

https://doi.org/10.26043/GISo.2020.1.6 


\section{REZENSION ZU: REIMER, DANIELA (HRSG.) (2019): SOZIALPÄDAGOGISCHE BLICKE. WEINHEIM/BASEL: BELTZ JUVENTA, 316 SEITEN, ISBN: 978-3-7799-6123-9, 34,95 EURO}

\section{Rebecca Mörgen}

Die Auseinandersetzung mit wie auch die Rede von einem "sozialpädagogischen Blick" ist eigentlich kein Novum. Doch: Was genau ist das charakteristische - gar spezifische - an einem sozialpädagogischen Blick? Mit dieser Frage beschäftigt sich der Sammelband, der Klaus Wolf anlässlich seiner Emeritierung gewidmet ist.

Der Sammelband gliedert sich neben einer Einleitung, einem Gespräch zwischen Sabine Hering und Klaus Wolf sowie Empfehlungen für eine Seniorprofessur in fünf Themenbereiche, die sozialpädagogische Handlungs- und Forschungsfelder darstellen - gefolgt wird hierbei dem sozialpädagogischen Blick von Klaus Wolf selbst (10). Insgesamt verfügt der Sammelband über 21 Beiträge und 29 Autor*innen, die als wissenschaftliche Wegbegleiter*innen die Denkbewegungen von Wolf (kritisch) würdigen.

Der erste Buchteil widmet sich mit drei Beiträgen dem Feld der Heimerziehung. Während Werner Freigang Wolfs Forschungslinien und disziplinwie professionspolitischen Ansätze in der Forschung wie auch Praxis der Heimerziehung skizziert, zeichnet Norbert Struck die Institutionengeschichte des Kinderheims „Rittmarshausen" nach. Der nachfolgende Beitrag (Theile) stellt die qualitative Analyse sozialer Netzwerkbeziehungen in der Biographie der Jugendlichen ins Zentrum. Netzwerkarbeit und die Bedeutsamkeit sozialer Netzwerke werden resümierend als "grundlegender Gelingensfaktor", „als Qualitätsmerkmal“ (61) für die Heimerziehung und Hilfeplanung betrachtet und für die Praxis eingefordert, um die Entwicklungsprozesse von Kindern und Jugendlichen begünstigend zu unterstützen.
Angefangen mit einem "Grundlagentext" (67), der die Entwicklungen der sozialpädagogischen Familienhilfe in Deutschland skizziert, werden die Leser*innen in sozialpädagogische Blickweisen auf familienunterstützende Arrangements eingeführt, die im zweiten Teil des Buches enthalten sind. Das Verhältnis von Therapie und Sozialer Arbeit steht im Zentrum des Beitrags von Andres Gut, wobei die Frage aufgeworfen wird, inwiefern sich ein sozialpädagogischer Blick mit einem therapeutischen Blick verbinden ließe (80). Anhand einer empirischen Fallsequenz sucht er diese Verhältnisbestimmung neu zu justieren. Lienhart und Hofer fokussieren insbesondere institutionelle Unterstützungsmöglichkeiten bei Rückkehrprozessen der Jugendlichen nach der Fremdunterbringung sowie das Verhältnis von ambulanter und stationärer Hilfe in Österreich.

Da das Buch Klaus Wolf gewidmet ist, mag es nicht verwundern, dass der dritte Teil, der sich der Pflegekinderhilfe widmet, der umfangreichste ist. Neben einem sehr persönlichen Rückblick auf die Gründung des „International Foster Care Research Network" durch Roger Bullock finden sich fünf weitere Beiträge. Der Beitrag von Corinna Petri setzt sich in konzeptioneller Absicht mit Zugehörigkeitskonstruktionen von Pflegekindern auseinander; problematisiert eine bislang eher "umgangssprachliche“ (118) Verwendung des Begriffs „Zugehörigkeit” in der Pflegekinderforschung und sucht theoretische Anknüpfungspunkte in der Sozialanthropologie und erziehungswissenschaftlichen Migrationsforschung. Während Yvonne Gassmann empirisch fundiert das Phänomen der „erworbenen Elternschaft" und der damit verbundenen spezifischen Verletzbarkeit in den Blick nimmt, fokussiert der praxisnahe Beitrag von Dirk Schäfer auf 
die leiblichen Eltern von Pflegekindern. Zentrale Kritik und Forderung des Autors an die Praxis ist, die jeweils divergierenden aber am Pflegeverhältnis beteiligten Perspektiven der Erwachsenen konsequent mit einzubeziehen und die Arbeit mit den Herkunftseltern zu intensivieren. Der Beitrag von Ehlke, Koch, Thomas und Schröer widmet sich der Frage, inwiefern „Jugend" in der Vollzeitpflege ermöglicht werden könne (131), und plädiert für den Begriff „Kinder und Jugendliche in Pflegeverhältnissen" (125). Über „(Pflege-)Familie“ denkt Michael Winkler in gegenstandstheoretischer Hinsicht nach. Er skizziert hierbei als ein Strukturmerkmal familiärer Lebensformen die „Krise als praktische Normalität" (156), hinterfragt in reflexiver Weise in der Pflegefamilienforschung etablierte Konzepte, wie die „Als-ob-Familie“ und blickt kritisch auf Professionalisierungsbestrebungen von Pflegefamilien, die diese eigentlich bedrohe, da Familien, so Winkler, „kaum als Reparaturorte“ (161) taugen. Mit einer an Wolf anknüpfenden „Perspektive der Reflexion" rückt er die Anerkennung familiärer Eigensinnigkeiten ins Zentrum.

Im vierten Teil fokussiert der „sozialpädagogische Blick" auf den Theorie-Praxis-Transfer. Zwei Beiträge beschäftigen sich mit Modell- und Praxisforschungsprojekten in der Pflegekinderhilfe und der Forschung zu Rückführungsprozessen in der Heimerziehung. Der dritte Beitrag ist von ehemaligen Studierenden verfasst und lässt sich als Reflexion der eigenen professionellen Haltung in der sozialpädagogischen Praxis verstehen. Lesenswert machen diesen Teil vor allem die Einblicke in konkrete Praxisforschungsprojekte, welche verschiedene involvierte professionelle Perspektiven berücksichtigen und aufzeigen, wie Kooperationen von Praxis und Wissenschaft erarbeitet wurden.

Zu guter Letzt wird der sozialpädagogische Blick auf die Disziplin der Sozialpädagogik selbst gerichtet. Eröffnet wird der Buchteil durch eine Auseinandersetzung mit der sozialpädagogischen Dimension von Partizipation (Gabriel/Tausendfreund). Theoretisch-informiert wird anhand eines Fallbeispiels aufgezeigt, inwiefern die Beteiligung an Entscheidungen, welche die Adressat*innen der Jugendhilfe selbst betreffen, Voraussetzung von Erziehungs- und Bildungsprozessen ist. Während Rhormann sich mit dem
Verhältnis von Sozialpädagogik und Sonderpädagogik in Bezug auf die Kinder- und Jugendhilfe beschäftigt, diskutiert Huisinga disziplinäre Berührungspunkte zwischen der Berufs- und Sozialpädagogik. Thomas Coelen skizziert anschließend „Veruneinseitigungen“ (274) von Bestimmungsversuchen dessen, was Sozialpädagogik als akademische Disziplin sei. Einen theoretisch-rekonstruktiven „Blick auf den sozialpädagogischen Blick" verfolgt Bernd Dollinger in seinem Beitrag. Zum einen wird der sozialpädagogische Blick als professions- und disziplinpolitisches Projekt rekonstruiert, das der kollektiven Identitätsbildung diene. Zum anderen wird eine Umkehrung sozialpädagogischer Blickverhältnisse gefordert: Denn die Rede von „dem“ sozialpädagogischen Blick bezieht sich auf die Konstitution und Konstruktion eines sozialpädagogischen Problems, das (vereindeutigende) Kategorisierungen und normative Vorannahmen über gesellschaftliche Deutungen von sozialpädagogischen Adressat*innen immer schon mit sich trage und die es reflexiv einzufangen gelte. Den Abschluss bildet ein kreativer Beitrag von Christian Niemeyer, der sich mit Nietzsche, Trump und dem Amokläufer Robert Steinhäuser auf die Couch legt und in fragender Form, die „stillen Gedanken“ (289) der Lesenden antizipierend, der Bedeutung eines sozialpädagogischen Verstehens nachgeht.

Dieses Buch ist ein besonderes und es ist ein Sammelband im besten Sinne. Besonders ist es, weil mit dieser Festschrift Weggefährt*innen inre Hochschätzung für Person und Werk eines "Grenzgängers" innerhalb der Sozialpädagogik als akademische Disziplin und als praxisbezogene Profession zum Ausdruck gebracht haben. Das in der Einleitung formulierte Ziel, ein Forschungs- und Lehrprogramm, das sich an der Schnittstelle von Wissenschaft und Praxis bewegt, zu skizzieren, gelingt dem Sammelband. Als roter Faden zieht sich die Rede von dem „sozialpädagogischen Blick" mal als Ausdruck einer spezifisch professionellen - und erlernten, ja verinnerlichten (167) - Haltung, mal als Phänomen und Gegenstand theoretisch-empirischer Überlegungen durch das Buch. Auf ganz unterschiedliche Art und Weise knüpfen die Beiträge dabei an Wolfs Überlegungen zu pädagogischen Prozessen in gesellschaftlichen Kontexten wie auch der Anerkennung der jeweiligen Subjektivität 
von Adressat*innen sozialpädagogischer Unterstützungs- und Hilfsangebote vor dem Horizont ihrer jeweiligen, ganz eigensinnigen biografischen Entwicklung an. Sie denken diese weiter, fordern an einzelnen Stellen zur Diskussion auf und regen dazu an, den Sammelband am Ende nochmals „gegen den Strich“ zu lesen, um die Spurensuche nach sozialpädagogischen Blickverhältnissen aufzunehmen. Doch diese Kritik verweist gleichzeitig auf eine Stärke des Bandes, sofern sich „der“ sozialpädagogische Blick nicht eindeutig bestimmen lässt und die Leser*innen zur Reflexion sozialpädagogischer Prozesse, der institutionellen Bearbeitung von subjektiven Lebensführungsweisen und etablierten Konzepten innerhalb der Sozialpädagogik als Disziplin und Profession einlädt.

\section{Zur Autorin}

Rebecca Mörgen ist wissenschaftliche Mitarbeiterin im Forschungsprojekt „Unbegleitete minderjährige Geflüchtete in institutioneller Betreuung: Chancen und Herausforderungen" (NFP 76) am Lehrstuhl für Ausserschulische Bildung und Erziehung am Institut für Erziehungswissenschaft der Universität Zürich. Sie forscht im Bereich sozialwissenschaftlicher Körper- und Leibforschung, phänomenologischer Erziehungswissenschaft, qualitativ-empirischer Migrations-, Ungleichheits- und Professionsforschung im Kontext Sozialpädagogik, Methoden qualitativ-rekonstruktiver Sozialforschung, insbesondere Ethnographie, Geschlechterforschung sowie zu Fragen sozialer Teilhabemöglichkeiten.

\section{Kontakt}

Dr. Rebecca Mörgen

Institut für Erziehungswissenschaft

Universität Zürich

Freiestr. 36

$\mathrm{CH}-8032$ Zürich

Tel.: +41446344563

E-Mail: rmoergen@ife.uzh.ch

URL: https://www.ife.uzh.ch/de/research/abe/mitarbeitende2/moergenrebecca.html 